\title{
Dynamics of electron-emission currents in plasmonic gaps induced by strong fields
}

\author{
Garikoitz Aguirregabiria, ab Dana-Codruta Marinica, ${ }^{c}$ Markus Ludwig, ${ }^{d}$ \\ Daniele Brida, de Alfred Leitenstorfer, J Javier Aizpurua iD *ab \\ and Andrey G. Borisov (iD *c
}

The dynamics of ultrafast electron currents triggered by femtosecond laser pulse irradiation of narrow gaps in a plasmonic dimer is studied using quantum mechanical Time-Dependent Density Functional Theory (TDDFT). The electrons are injected into the gap due to the optical field emission from the surfaces of the metal nanoparticles across the junction. Further evolution of the electron currents in the gap is governed by the locally enhanced electric fields. The combination of TDDFT and classical modelling of the electron trajectories allows us to study the quiver motion of the electrons in the gap region as a function of the Carrier Envelope Phase (CEP) of the incident pulse. In particular, we demonstrate the role of the quiver motion in establishing the CEPsensitive net electric transport between nanoparticles.

\section{Introduction}

Electron emission from metallic surfaces is a physical process which exploits the exchange of energy between incident photons and outgoing electrons. ${ }^{1,2}$ The current trends in nanotechnology are able to design complex morphologies of metallic nanostructures which can act as effective optical nanoantennas trapping and enhancing light of specific wavelengths in their proximity ${ }^{3-5}$ This is achieved by means of the collective excitation of the electronic charge density at the interfaces of the metallic nanostructures, so-called surface plasmons. ${ }^{6,7}$ Plasmonic nanoparticles are thus a very appealing structure for the generation of strong-field emitted electrons due to the electric-field enhancement and

${ }^{a}$ Centro de Física de Materiales CSIC-UPV/EHU, Paseo Manuel de Lardizabal 5, 20018, Donostia-San Sebastián, Spain.E-mail: aizpurua@ehu.eus

${ }^{b}$ Donostia International Physics Center, Paseo Manuel de Lardizabal 4, 20018, Donostia-San Sebastián, Spain 'Institut des Sciences Moléculaires d'Orsay - UMR 8214, CNRS-Université Paris Sud, Batiment 520, 91405 Orsay Cedex, France.E-mail: andrei.borissov@u-psud.fr

${ }^{d}$ Department of Physics, Center for Applied Photonics, University of Konstanz, D-78457 Konstanz, Germany ${ }^{e}$ Physics \& Materials Science Research Unit, University of Luxembourg, 162a Avenue de la Faïencerie, L-1511, Luxembourg 
localization produced near the particle surface. ${ }^{8-15}$ Among others, metallic tips have been commonly used in the generation of photoemitted electrons, for instance in the guns of electron microscopes. ${ }^{16}$

More recently, plasmonic gaps, formed in a metal-insulator-metal junction, have been shown to host very intense bonding-gap plasmons which produce electromagnetic hot spots commonly exploited in surface-enhanced spectroscopy. ${ }^{17-22}$ The plasmonic gap has also been the object of intense research regarding the interplay between optical and transport properties, as the two metallic particles forming the gap can be externally biased, and the current across the gap can thus be accessed while illuminating the structure with light. ${ }^{22-27}$ Last, but not least, the ability to shape light into ultra-short single-cycle pulses with varying Carrier Envelope Phase (CEP) provides an additional degree of control on the photoelectrons emitted., ${ }^{9,13,24}$ When those electrons are emitted in a plasmonic gap as a result of the incidence of an ultra-short laser pulse, a complex dynamical behaviour of the electrons can be foreseen which depends on the particular conditions of the emission, such as energy of the photons, duration of the pulse, local field enhancement, or size of the gap. ${ }^{24}$ Differently from a metallic tip, characterized by electron emission into free-space, the plasmonic gap offers the possibility of achieving electron transport between nanoparticles controlled at the optical cycle time-scale thus granting mutual coherence between radiation and electron current. A theoretical scheme capable of tracing the dynamics of these ultra-short electron bursts in plasmonic gaps can be extremely useful when it comes to the design and control of the optoelectronic properties of ultrafast electron currents.

This discussion precisely addresses the challenge of describing the complex dynamics of photoemitted currents in plasmonic gaps under ultra-short illumination. A simple model of the structure will help to provide an understanding of the interplay between the carrier envelope phase of a single pulse and the motion of the electron bursts in the gap. We describe our model system, the methodological approach to the dynamics of the electrons, and the results in the following sections.

\section{Plasmonic system}

As a prototype of a plasmonic gap system we consider a dimer of parallel gold cylinders of diameter $D=10 \mathrm{~nm}$ as sketched in Fig. 1 . The identical cylinders are infinite along the $z$-axis and the distance between the two nanoparticle surfaces at the closest point is $d_{\text {gap }}=3 \mathrm{~nm}$ as measured along the $x$-axis. The centre of the gap is located at $(x=0, y=0)$. This structure is illuminated by a single-cycle femtosecond (fs) Gaussian pulse polarized along the $x$-axis. The electric field of the pulse is given by:

$$
E(t)=\tilde{E} \cos (\omega t+\phi) \mathrm{e}^{-t^{2} / \tau^{2}},
$$

where the amplitude of the electric field is $\tilde{E}=3.7 \mathrm{~V} \mathrm{~nm}^{-1}$, $\omega$ is the carrier frequency, $\phi$ is the CEP, and $\tau=(2 \pi / \omega) / \sqrt{2 \log (2)}$ is the duration of the pulse. Fig. 1 shows two of the pulse CEPs used in the calculation: in blue $\phi=0$ and in red $\phi=\pi / 2$. We use carrier frequencies $\omega$ in the near-infrared, thus far from the bonding dipolar plasmon resonance of the dimer at $\Omega_{\mathrm{p}} \cdot{ }^{25,28}$ With the present 


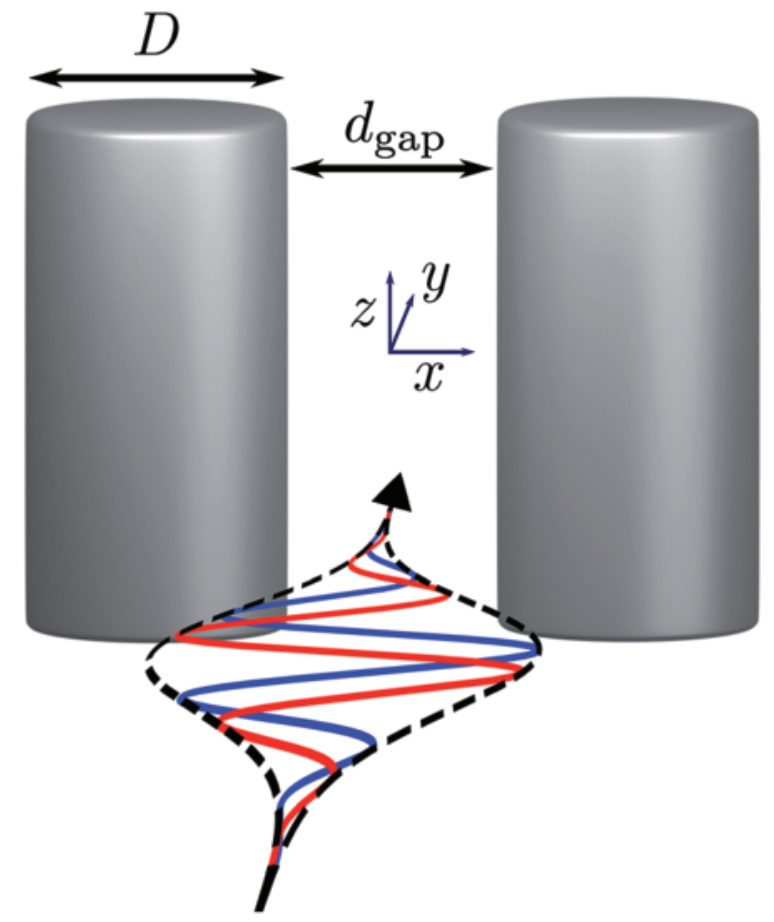

Fig. 1 Dimer of parallel gold cylinders of diameter $D=10 \mathrm{~nm}$. The cylinders are separated by a gap of $d_{\text {gap }}=3 \mathrm{~nm}$. The dimer is excited by a sub-cycle laser pulse with a Gaussian envelope characterized by a carrier frequency $\omega$ and a carrier envelope phase $\phi$. Two examples of the laser electric field are shown for $\phi=0$ (blue line) and $\phi=\pi / 2$ (red line). We study the dynamics of the optical-field emitted electrons in the plasmonic gap and their dependence on $\phi$ and $\omega$.

choice of model to describe the electronic structure of the gold cylinders, as discussed below, $\Omega_{\mathrm{p}}=5.5 \mathrm{eV}$. Due to the off-resonance excitation of the system the time-dynamics of the local fields in the gap are similar to those of the incident pulse (sub-cycle). Since the local field in the gap triggers the electron current across the junction it is expected that the net electronic transport will be sensitive to the CEP of the external pulse. Contrary to this situation, for the resonant excitation the dynamics of the field in the junction would be determined by the damping rate of the corresponding plasmon mode. As a result, except for an extremely broad plasmon resonance, the local ac field in the gap would generally last a long time after the end of the external transient. In such a resonant situation, the averaging by many-cycle oscillations would lead to an almost zero net current across the junction and very low sensitivity to the CEP of the incident pulse.

Under these off-resonance illumination conditions, with the corresponding field enhancement in the gap, and the range of frequencies used, the Keldish parameter $^{29}(\gamma)$ obtained in this work is in the range of $\gamma \approx 0.48-1.90$, which is usually considered within the optical-field emission regime..$^{2,8,30,31}$

\section{Methodology}

To treat the electron dynamics of the system under strong-field illumination we use Time-Dependent Density Functional Theory (TDDFT) based on the KohnSham (KS) scheme. ${ }^{32-34}$ The Au cylinders are described with the so-called 
Stabilized Jellium Model (SJM) ${ }^{35}$ Within the SJM, the valence electrons are treated explicitly, while the atomic cores are represented as a positive background charge density

$$
n^{+}=\left(\frac{4 \pi}{3} r_{\mathrm{s}}^{3}\right)^{-1}
$$

where $r_{\mathrm{s}}$ is the Wigner-Seitz radius (here $r_{\mathrm{s}}=3.02 a_{0}, 1 a_{0}=0.053 \mathrm{~nm}$ ). An attractive pseudopotential ( $V_{\text {st }}$, see below) is used so that the empirical work function of $\mathrm{Au}^{36}, \Phi=5.5 \mathrm{eV}$, is retrieved with the ground-state Density Functional Theory (DFT) ${ }^{32}$ calculations. Even though the use of the SJM neglects atomistic details and effects linked with localized d-electron bands, it allows us to trace the dynamics of valence electrons in the metal, which are involved in the screening, photoemission, strong-field ionization and transport properties in the plasmonic dimer. The jellium model of metal nanoparticles has been successfully used in the context of plasmonics allowing the theoretical prediction of quantum effects later confirmed experimentally ${ }^{23,37,38}$ as well as corroborated by TDDFT studies of strong-field emission from metal nanotips. ${ }^{39}$

The time evolution of the KS orbitals is obtained from the 2D time-dependent KS equations (all equations are written in atomic units, a.u., unless otherwise indicated):

$$
\left[-\frac{1}{2} \nabla^{2}+V_{\text {eff }}[n](x, y, t)\right] \Psi_{\mathrm{k}}(x, y, t)=i \frac{\partial}{\partial t} \Psi_{\mathrm{k}}(x, y, t)
$$

where $\Psi_{\mathrm{k}}$ are the KS orbitals, $\nabla=\hat{x} \frac{\partial}{\partial x}+\hat{y} \frac{\partial}{\partial y}$, where $\hat{x}(\hat{y})$ is the unit length vector in the direction of the $x(y)$ axis. The effective KS potential $V_{\text {eff }}[n](x, y, t)$ is given by:

$$
\mathrm{V}_{\mathrm{eff}}[n](x, y, t)=V_{\mathrm{H}}[n](x, y, t)+V_{\mathrm{xc}}[n](x, y, t)+V_{\mathrm{st}}(x, y, t)+V_{\mathrm{ext}}(x, t) .
$$

The Hartree potential, $V_{\mathrm{H}}[n](x, y, t)$, accounts for the interaction with the charge density of the system. Due to the subwavelength scale of the relevant dimensions of the cylindrical dimer, the non-retarded approximation is considered in the calculations. The exchange-correlation potential, $V_{\mathrm{xc}}[n](x, y, t)$, effectively incorporates the exchange and correlation effects of the many-electron interacting system. Here we use the functional of O. Gunnarsson and B. I. Lundqvist ${ }^{40}$ within the Adiabatic Local Density Approximation (ALDA). ${ }^{34,41}$ The stabilizing potential, $V_{\mathrm{st}}(x, y, t)$, corresponds to an attractive constant potential in the region of space filled by the nanoparticles, allowing the correct work function of gold to be obtained as already discussed above. Finally, $V_{\text {ext }}(x, t)=x E(t)$ is the interaction potential between an electron and the external electromagnetic field.

The Fourier-grid Hamiltonian approach ${ }^{42-45}$ with wavefunction representation on a grid of equidistant points in $x$ - and $y$-coordinates, and the Split-Operator technique ${ }^{43,46,47}$ are used to solve eqn (3) by short time-step propagation. The initial conditions are $\Psi_{\mathrm{k}}(x, y, t=0)=\Psi_{\mathrm{k}}{ }^{0}(x, y)$, where $\Psi_{\mathrm{k}}{ }^{0}$ correspond to the ground state KS orbitals retrieved from self-consistent DFT $^{32}$ calculation of the system in the absence of the external electromagnetic field.

The time-dependent electronic density and electron current density, $n(x, y, t)$ and $\vec{j}(x, y, t)$ respectively, are computed at each time step as follows: 


$$
\begin{gathered}
n(x, y, t)=2 \sum_{\mathrm{k} \in \text { occ }} \chi_{\mathrm{k}}\left|\Psi_{\mathrm{k}}(x, y, t)\right|^{2}, \\
\vec{j}(x, y, t)=2 \sum_{\mathrm{k} \in \text { occ }} \chi_{\mathrm{k}} \operatorname{Re}\left[\Psi_{\mathrm{k}}^{*}(x, y, t) \hat{\mathbf{p}} \Psi_{\mathrm{k}}(x, y, t)\right],
\end{gathered}
$$

where $\hat{\mathbf{p}}=-i \nabla$, the sum runs over all the occupied KS orbitals, the factor 2 accounts for the spin degeneracy, and $\chi_{\mathrm{k}}=\frac{1}{\pi} \sqrt{2\left(E_{\mathrm{F}}-E_{\mathrm{k}}\right)}$ accounts for the number of electronic states associated with the z-motion. $E_{\mathrm{F}}$ stands for the Fermi level of the nanostructures and $E_{\mathrm{k}}$ for the ground-state energy of the orbital k.

\section{Results}

To study the dynamics of strong-field emitted electrons in the gap, we illuminate the system with the field transient given by eqn (1) mimicking a single-cycle optical pulse, and at each time step we compute the electron current across the mid-plane of the gap,

$$
J(x=0, t)=\int_{-\infty}^{\infty} \hat{x} \vec{j}(x=0, y, t) \mathrm{d} y .
$$

Fig. 2 shows the result of such analysis for the case of a pulse carrier frequency $\omega=0.67 \mathrm{eV}$, where the electric field at the centre of the gap, $E_{\text {gap }}$, is plotted in blue and the current density across the mid-plane of the gap, $J(x=0, t)$, is plotted in red. $E_{\text {gap }}$ comprises both the electric field of the incident pulse and the induced electric field due to the polarisation of the nanowires. Prior to the central peak of the pulse at $t=0$, the electromagnetic field in the junction is not strong enough to produce a significant electron current through the middle of the gap. Upon arrival, the central peak produces the emission of electrons from the surface of the cylinder on the right hand side in Fig. 1 and therefore a negative electronic current is observed at $t \approx 1 \mathrm{fs}$. Around $t \approx 1.5 \mathrm{fs}$, the field in the gap changes polarity and a second smaller current density peak can be observed, which flows in the opposite direction to the main one. This weaker current density is formed

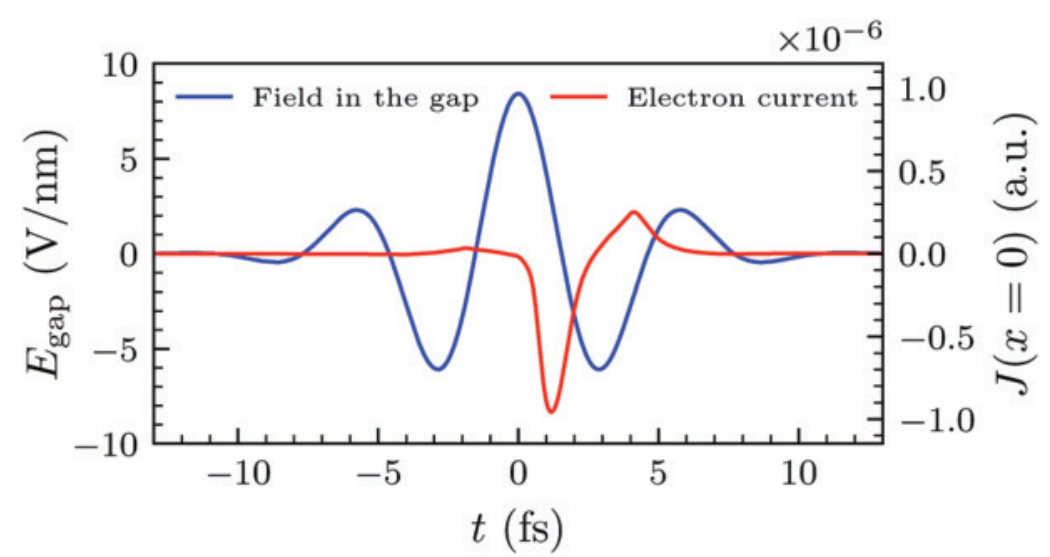

Fig. 2 TDDFT results for the dimer illuminated by a pulse of carrier frequency $\omega=0.67 \mathrm{eV}$ and CEP $\phi=0$. Blue line: electric field at the centre of the gap, $E_{\text {gap. }}$ Red line: electron current across the mid-plane of the gap, $J(x=0, t)$. 
from two contributions. The first contribution is due to the cylinder situated on the left hand side in Fig. 1. The second contribution, as we will show below, is originating from electrons which were emitted from the right cylinder but did not reach the opposite nanoparticle before $E_{\text {gap }}$ changed polarity, thus inducing electron quiver motion.

Integrating in time the current in eqn (7), one can obtain the number of electrons transferred across the gap. The analysis of the net electron transport as a function of CEP is shown in Fig. 3 for different carrier frequencies of the incident pulse. We first focus our attention on the lowest frequencies, $\omega=0.32 \mathrm{eV}$ (solid grey line) and $\omega=0.48 \mathrm{eV}$ (solid pink line). For these frequencies, the results in Fig. 3 show a behaviour consistent with the direct propagation of the emitted electrons across the junction without any effect of the quiver motion. ${ }^{\mathbf{1 0 - 1 2}}$ Indeed, for a CEP $\phi=\pi / 2$ or $\phi=3 \pi / 2$, the local field in the gap, $E_{\text {gap }}$, is antisymmetric (sine-like) with respect to the centre of the Gaussian envelope and thus, the field shows two identical peaks of opposite polarity (see the red line in the sketch of Fig. 1). In such a situation, the amount of electrons transferred in both directions is equal, they compensate each other, and the net transport is zero. The maximum electron transfer occurs for $\phi=0$ or $\phi=\pi$. The incident electromagnetic pulse in this case has a symmetric electric field profile, with the main positive or negative peak centred at $t=0$ (see Fig. 2). As the optical-field emission process is highly non-linear, ${ }^{\mathbf{3 0}, 31}$ the electron current in the junction is dominated by the ultra-short burst of electrons emitted at the half-period with strongest field, as nicely observed in Fig. 2. Under the assumption that all the electrons arrive to the opposite surface, $\phi=0$ or $\phi=\pi$ correspond to the

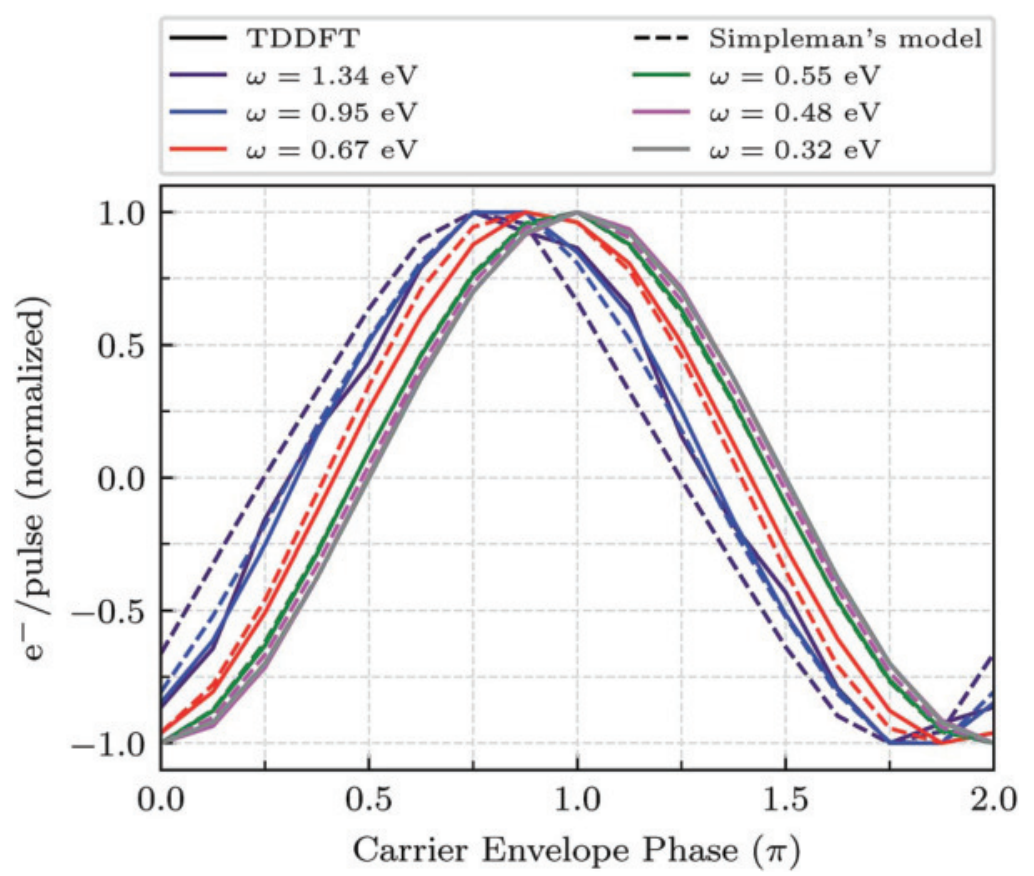

Fig. 3 Normalized electron transfer per pulse as a function of the CEP $\phi$. Different colours indicate the various carrier frequencies considered. Solid lines: results of the TDDFT calculations. Dashed lines: results obtained using Simpleman's model for electron emission and transport. The quiver amplitude associated to each of the frequencies ( $\omega=0.32 \mathrm{eV}, 0.48 \mathrm{eV}, 0.55 \mathrm{eV}, 0.67 \mathrm{eV}, 0.95 \mathrm{eV}$ and $1.34 \mathrm{eV}$ ) is $X_{\mathrm{q}}=6.33 \mathrm{~nm}, 2.82 \mathrm{~nm}$, $2.12 \mathrm{~nm}, 1.42 \mathrm{~nm}, 0.72 \mathrm{~nm}$ and $0.37 \mathrm{~nm}$, respectively. 
maximum number of electrons transferred in positive $(\phi=\pi)$ or negative $(\phi=0)$ directions of the $x$ axis.

However, when the illumination frequency is increased, the Carrier Envelope Phase (CEP) at which the maximum electron transfer is produced shifts away from the expected $\phi=0$ and $\phi=\pi$ values. In contrast to single nanoparticles where the evanescent near-field decays rapidly as the distance to the nanoparticle's surface is increased and the quiver motion might be quenched, ${ }^{10}$ the field in small plasmonic gaps is almost constant across the full distance separating the nanoparticles. Therefore, the quiver motion of the emitted electrons needs to be carefully considered. This quiver motion of the electrons in the gap is responsible for the shift of the value of the CEP corresponding to the maximum net electron current shown in Fig. 3, as we analyse below.

\section{Classical analysis}

We employ the classical Simpleman's model (SMM) to study the quiver motion of the photoemitted electrons in the plasmonic gap, and the role that the quiver motion plays in the electron transfer between the nanoparticles. The SMM has been first developed to describe the optical field electron emission from atomic species, ${ }^{48-50}$ and later successfully applied to electron emission from surfaces of metallic tips. ${ }^{30,31,39}$ To qualitatively capture the main physical effects, while keeping the analysis of the results simple, we neglect the 2D aspect of the problem and only consider the electron motion along the $x$-axis. The optical field electron emission is treated as a two step process. First we use the field in the gap, $E_{\text {gap }}$, obtained from the TDDFT calculations to compute the instantaneous FowlerNordheim tunneling current, ${ }^{51} J_{\mathrm{FN}}$, at each time step within the pulse,

$$
J_{\mathrm{FN}} \propto-E_{\text {gap }}\left(t_{\mathrm{e}}\right)\left|E_{\text {gap }}\left(t_{\mathrm{e}}\right)\right| \exp \left(-\frac{b \Phi^{\frac{3}{2}}}{\left|E_{\text {gap }}\left(t_{\mathrm{e}}\right)\right|}\right),
$$

where $b=6.83 \mathrm{~V}\left(\mathrm{~nm}^{-1} \mathrm{eV}^{-3 / 2}\right), \Phi=5.5 \mathrm{eV}$ is the work function of gold, and $E_{\text {gap }}\left(t_{\mathrm{e}}\right)$ is the electric field in the gap at the time instant of emission defined as $t_{\mathrm{e}}$. The second step consists of calculating the classical trajectory on the $x$-axis of an electron emitted at time $t_{\mathrm{e}}$ and subjected to the time-dependent electric field $E_{\text {gap }}$ (assuming that $E_{\text {gap }}$ is homogeneous in the gap region):

$$
\dot{v}_{\mathrm{x}}=-E_{\text {gap }}
$$

The initial condition $v_{\mathrm{x}}\left(t_{\mathrm{e}}\right)=0$ corresponds to a tunneling electron emerging from the potential barrier at the metal/vacuum interface into the classically allowed region in the gap. Using the relationship between the electric field and the vector potential $(A), E=-\mathrm{d} A / \mathrm{d} t$, the velocity of the classical electron at any time $t>t_{\mathrm{e}}$ is:

$$
v_{\mathrm{x}}(t)=A_{\text {gap }}(t)-A_{\text {gap }}\left(t_{\mathrm{e}}\right) .
$$

This trajectory is computed until the electron reaches the surface of one of the two cylinders. If the electron crosses the gap, the trajectory is considered for the calculation of the net charge transfer assuming that it contributes with a weight given by eqn (8). The trajectory is discarded if, because of the quiver motion, the 
electron returns back to the initial nanoparticle. We thus neglect the possible, albeit small, contribution of the electron back-scattering. ${ }^{39}$

Analysing eqn (10), a third scenario is possible. If an electron is emitted at a time $t_{\mathrm{e}}$ such that $E_{\text {gap }}$ is a local maximum, the associated vector potential is $A_{\text {gap }}\left(t_{\mathrm{e}}\right)=0$. Furthermore, the finite duration of the illumination transient ensures that $A_{\text {gap }}(t \rightarrow \infty)=0$. Therefore, electrons emitted within the condition $A_{\text {gap }}\left(t_{\mathrm{e}}\right)=0$ might end up in the middle of the gap with velocity $v_{\mathrm{x}}(t \rightarrow \infty)=0$. We will call these trajectories as "trapped" trajectories.

The electron transfer across the plasmonic gap calculated within the SMM as a function of the CEP and frequency of the incident pulse is shown with dashed lines in Fig. 3. The agreement with the TDDFT calculations (solid lines) is remarkable. The Simpleman's model allows the identification of the quiver amplitude $X_{\mathrm{q}}$ as a key parameter controlling the electron transfer. The quiver amplitude, $X_{\mathrm{q}}$, is the amplitude of the oscillatory movement of an electron in a homogeneous harmonic electric field, $E(t)=E_{0} \cos (\omega t)$, of frequency $\omega$ and amplitude strength $E_{0}$ :

$$
X_{\mathrm{q}}=\frac{E_{0}}{\omega^{2}}
$$

Taking $E_{0}$ as the maximum of the total electric field generated in the gap, $E_{0}=$ $\left[E_{\text {gap }}\right]_{\text {max }} \approx 8.45 \mathrm{~V} \mathrm{~nm}^{-1}$, the carrier frequencies of the incident electromagnetic pulse $\omega=0.32 \mathrm{eV}, 0.48 \mathrm{eV}, 0.55 \mathrm{eV}, 0.67 \mathrm{eV}, 0.95 \mathrm{eV}$ and $1.34 \mathrm{eV}$ result in the corresponding quiver amplitudes $X_{\mathrm{q}}=6.33 \mathrm{~nm}, 2.82 \mathrm{~nm}, 2.12 \mathrm{~nm}, 1.42 \mathrm{~nm}$, $0.72 \mathrm{~nm}$ and $0.37 \mathrm{~nm}$, respectively. As follows from the results presented in Fig. 3, the maximum electric transport is produced at CEP $\phi=0$ or $\phi=\pi$ only for the cases where $X_{\mathrm{q}} \gtrsim d_{\text {gap }}$.
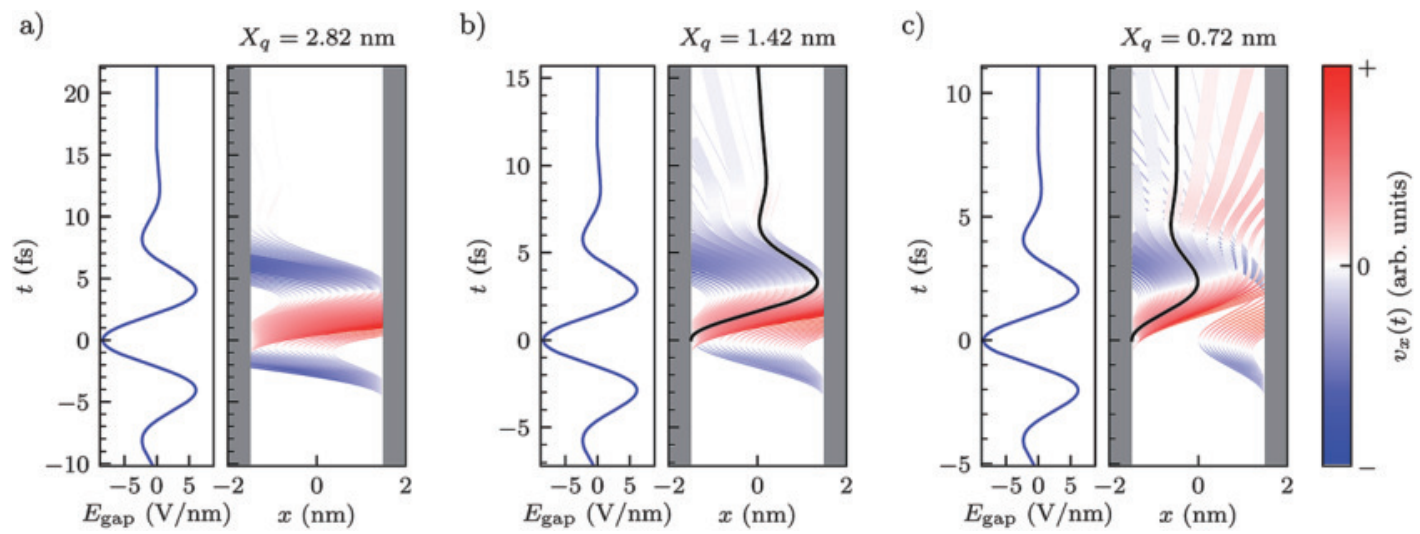

Fig. 4 Classical trajectories obtained using the Simpleman's model for carrier frequencies of the incident pulse (a) $\omega=0.48 \mathrm{eV}$, (b) $\omega=0.67 \mathrm{eV}$ and (c) $\omega=0.95 \mathrm{eV}$. In all cases the $\operatorname{CEP} \phi=\pi$. The electric field at the centre of the gap, $E_{\text {gap }}$ (horizontal axis) as a function of time (vertical axis) is shown to the left of each panel. The classical trajectories of electrons emitted along the $x$-axis are shown to the right of each panel. The position $x$ is shown on the horizontal axis and time on the vertical axis. The $x$-component of the velocity of the electrons in each trajectory is colour-coded with positive velocity in red and negative in blue. The velocity in each panel is normalized by the maximum velocity in the $x$-direction to the range $[-1,1]$. The weight of each trajectory as given by eqn (8) is represented by the width of the corresponding trajectory line. Wider lines imply a larger weight and thus a larger amount of charge moving along in such trajectory. The black lines in (b) and (c) mark two examples of "trapped" trajectories. 
Fig. 4 shows the electric field in the gap (left line-plot in each panel) and the color-map formed by classical electron trajectories (right side of each panel) for three different carrier frequencies selected in such a way that the respective quiver amplitudes belong to the two different regimes, i.e., $X_{\mathrm{q}} \gtrsim d_{\text {gap }}$ and $X_{\mathrm{q}}<d_{\text {gap }}$. In the three cases the CEP is $\phi=\pi$. The velocity of the electron in each trajectory is colour-coded, having positive velocity $\left(v_{\mathrm{x}}>0\right)$ marked in red and negative velocity $\left(v_{\mathrm{x}}<0\right)$ in blue. The width of each line is linked with the tunneling current in eqn (8), which indicates that a larger amount of charge is moving along wider lines. In the three examples quiver motion is observed depending on the moment $t_{\mathrm{e}}$ when the electrons are emitted. In Fig. $4 \mathrm{a}, X_{\mathrm{q}}=2.82 \mathrm{~nm}$ and thus, most of the electron trajectories reach the opposite nanoparticle before the ballistic motion is inverted. In particular it can be observed how almost all the trajectories involving a large number of emitted electrons (wider lines) directly reach the opposite nanoparticle, corroborating that the maximum net electron transfer occurs for $\phi=\pi$ (see the pink line in Fig. 3).

Fig. $4 \mathrm{~b}$ and $\mathrm{c}$ show the situation where $X_{\mathrm{q}}<d_{\text {gap }}$. In both cases the quiver motion is more obvious and relevant as $X_{\mathrm{q}}$ decreases. An increasing number of trajectories quiver back because the polarity of the field changes before they reach the opposite side of the gap. It is particularly relevant that in these two cases many of the trajectories involving a large number of emitted electrons (wide lines) are not able to cross the gap and thus the maximum electron transfer no longer occurs for the incident pulse with CEP $\phi=\pi$ which is the value displayed in this figure. This effect is consistent with the situation observed for larger illumination frequencies displayed with blue and red lines in Fig. 3. The black lines in Fig. 4b and c follow two examples of "trapped" trajectories where, as already mentioned, the final velocity is $v_{\mathrm{x}} \approx 0$. The electrons moving along "trapped" trajectories are thus stopped in the middle of the gap upon the termination of the electromagnetic pulse. In practice, these electrons will be absorbed by the substrate or deviated by the stray fields without contributing to the coherent charge transport. As follows from the discussion above, the classical SMM analysis turns out to be a powerful and intuitive tool to trace the dynamics of ultrafast electron currents in plasmonic gaps driven by light.

\section{Discussion and conclusion}

Using a combination of quantum calculations (TDDFT) and classical modelling (Simpleman's model) we have been able to address the complex dynamics of strong-field emission currents in plasmonic gaps. In contrast with the reported results using metallic tips, ${ }^{10,12}$ we observed that the homogeneous field distribution characteristic of small plasmonic gaps produces a situation where the quiver motion of electrons has a substantial impact on the electron current. The ratio between the electron quiver amplitude, $X_{\mathrm{q}}$, and the gap distance separating the nanoparticles, $d_{\text {gap }}$, is a key parameter to establish the absolute Carrier Envelope Phase (CEP) which produces the maximum electron transport between nanoparticles. These results reveal the importance of the CEP of an incident pulse to control the mutual coherence between electron and photon dynamics in specific metallic junctions. 


\section{Conflicts of interest}

There are no conflicts of interest to declare.

\section{Acknowledgements}

G. A. acknowledges project PI2017-30 of the Departamento de Educación, Política Lingüística y Cultura of the Basque government and, G. A. and J. A. acknowledge funding from project FIS2016-80174-P of the Ministry of Economy, Industry and Competitiveness MINEICO.

\section{Notes and references}

1 P. Dombi, S. E. Irvine, P. Rácz, M. Lenner, N. Kroó, G. Farkas, A. Mitrofanov, A. Baltuška, T. Fuji, F. Krausz and A. Y. Elezzabi, Opt. Express, 2010, 18, $24206-24212$.

2 S. V. Yalunin, M. Gulde and C. Ropers, Phys. Rev. B: Condens. Matter Mater. Phys., 2011, 84, 195426.

3 J. Aizpurua, G. W. Bryant, L. J. Richter, F. G. De Abajo, B. K. Kelley and T. Mallouk, Phys. Rev. B: Condens. Matter Mater. Phys., 2005, 71, 235420.

4 M. Pelton, J. Aizpurua and G. Bryant, Laser Photonics Rev., 2008, 2, 136-159.

5 L. Novotny and N. Van Hulst, Nat. Photonics, 2011, 5, 83-90.

6 S. A. Maier, Plasmonics: fundamentals and applications, Springer Science \& Business Media, 2007.

7 L. Novotny and B. Hecht, Principles of nano-optics, Cambridge university press, 2012.

8 R. Bormann, M. Gulde, A. Weismann, S. V. Yalunin and C. Ropers, Phys. Rev. Lett., 2010, 105, 147601.

9 M. Krüger, M. Schenk and P. Hommelhoff, Nature, 2011, 475, 78.

10 G. Herink, D. R. Solli, M. Gulde and C. Ropers, Nature, 2012, 483, 190.

11 D. J. Park, B. Piglosiewicz, S. Schmidt, H. Kollmann, M. Mascheck and C. Lienau, Phys. Rev. Lett., 2012, 109, 244803.

12 P. Dombi, A. Hörl, P. Rácz, I. Márton, A. Trügler, J. R. Krenn and U. Hohenester, Nano Lett., 2013, 13, 674-678.

13 B. Piglosiewicz, S. Schmidt, D. J. Park, J. Vogelsang, P. Groß, C. Manzoni, P. Farinello, G. Cerullo and C. Lienau, Nat. Photonics, 2014, 8, 37.

14 M. Lehr, B. Foerster, M. Schmitt, K. Krüger, C. Sönnichsen, G. Schönhense and H.-J. Elmers, Nano Lett., 2017, 17, 6606-6612.

15 W. P. Putnam, R. G. Hobbs, P. D. Keathley, K. K. Berggren and F. X. Kärtner, Nat. Phys., 2017, 13, 335.

16 A. V. Crewe, D. N. Eggenberger, J. Wall and L. M. Welter, Rev. Sci. Instrum., 1968, 39, 576-583.

17 P. Nordlander, C. Oubre, E. Prodan, K. Li and M. I. Stockman, Nano Lett., 2004, 4, 899-903.

18 P. Mühlschlegel, H.-J. Eisler, O. J. F. Martin, B. Hecht and D. W. Pohl, Science, 2005, 308, 1607-1609.

19 I. Romero, J. Aizpurua, G. W. Bryant and F. J. G. De Abajo, Opt. Express, 2006, 14, 9988-9999.

20 H. Xu, E. J. Bjerneld, M. Käll and L. Börjesson, Phys. Rev. Lett., 1999, 83, 4357. 
21 S. S. Acimovic, M. P. Kreuzer, M. U. González and R. Quidant, ACS Nano, 2009, 3, 1231-1237.

22 M. Liao, S. Jiang, C. Hu, R. Zhang, Y. Kuang, J. Zhu, Y. Zhang and Z. Dong, Nano Lett., 2016, 16, 4040-4046.

23 K. J. Savage, M. M. Hawkeye, R. Esteban, A. G. Borisov, J. Aizpurua and J. J. Baumberg, Nature, 2012, 491, 574-577.

24 T. Rybka, M. Ludwig, M. F. Schmalz, V. Knittel, D. Brida and A. Leitenstorfer, Nat. Photonics, 2016, 10, 667.

25 D. C. Marinica, M. Zapata, P. Nordlander, A. K. Kazansky, P. M. Echenique, J. Aizpurua and A. G. Borisov, Sci. Adv., 2015, 1, e1501095.

26 D. R. Ward, F. Hüser, F. Pauly, J. C. Cuevas and D. Natelson, Nat. Nanotechnol., 2010, 5, 732.

27 T. L. Cocker, V. Jelic, M. Gupta, S. J. Molesky, J. A. J. Burgess, G. De Los Reyes, L. V. Titova, Y. Y. Tsui, M. R. Freeman and F. A. Hegmann, Nat. Photonics, 2013, 7, 620 .

28 G. Aguirregabiria, D. C. Marinica, R. Esteban, A. K. Kazansky, J. Aizpurua and A. G. Borisov, Phys. Rev. B, 2018, 97, 115430.

29 L. Keldysh, et al., J. Exp. Theor. Phys., 1965, 20, 1307-1314.

30 M. Krüger, M. Schenk, M. Förster and P. Hommelhoff, J. Phys. B: At., Mol. Opt. Phys., 2012, 45, 074006.

31 M. Krüger, C. Lemell, G. Wachter, J. Burgdörfer and P. Hommelhoff, J. Phys. B: At., Mol. Opt. Phys., 2018, 51, 172001.

32 W. Kohn and L. J. Sham, Phys. Rev., 1965, 140, A1133.

33 E. Runge and E. K. U. Gross, Phys. Rev. Lett., 1984, 52, 997.

34 M. A. Marques and E. Gross, Annu. Rev. Phys. Chem., 2004, 55, 427-455.

35 J. P. Perdew, H. Q. Tran and E. D. Smith, Phys. Rev. B: Condens. Matter Mater. Phys., 1990, 42, 11627.

36 E. V. Chulkov, V. M. Silkin and P. M. Echenique, Surf. Sci., 1999, 437, 330-352.

37 R. Esteban, A. G. Borisov, P. Nordlander and J. Aizpurua, Nat. Commun., 2012, 3, 825 .

38 J. A. Scholl, A. García-Etxarri, A. L. Koh and J. A. Dionne, Nano Lett., 2013, 13, 564-569.

39 M. Krüger, M. Schenk, P. Hommelhoff, G. Wachter, C. Lemell and J. Burgdörfer, New J. Phys., 2012, 14, 085019.

40 O. Gunnarsson and B. Lundqvist, Phys. Rev. B: Condens. Matter Mater. Phys., 1976, 13, 4274.

41 K. Burke, J. Werschnik and E. Gross, J. Chem. Phys., 2005, 123, 062206.

42 D. Kosloff and R. Kosloff, J. Comput. Phys., 1983, 52, 35-53.

43 R. Kosloff, J. Phys. Chem., 1988, 92, 2087-2100.

44 C. C. Marston and G. G. Balint-Kurti, J. Chem. Phys., 1989, 91, 3571-3576.

45 R. Kosloff, Dynamics of molecules and chemical reactions, 1996, pp. 185-230.

46 M. D. Feit, J. A. Fleck and A. Steiger, J. Comput. Phys., 1982, 47, 412-433.

47 M. D. Feit and J. A. Fleck Jr, J. Chem. Phys., 1983, 78, 301-308.

48 P. B. Corkum, Phys. Rev. Lett., 1993, 71, 1994.

49 G. G. Paulus, W. Becker, W. Nicklich and H. Walther, J. Phys. B: At., Mol. Opt. Phys., 1994, 27, L703.

50 G. Paulus, W. Becker and H. Walther, Phys. Rev. A: At., Mol., Opt. Phys., 1995, 52, 4043.

51 R. G. Forbes, Appl. Phys. Lett., 2006, 89, 113122. 